\title{
Was sagen Statistiken über Entgeltdiskriminierung aus?
}

\author{
Dr. Karin Tondorf \\ Freie Wissenschaftlerin und Beraterin, Seddiner See
}

In jüngster Zeit findet das Thema „Entgeltgleichheit zwischen Frauen und Männern“ größere Aufmerksamkeit in der Öffentlichkeit. Ausgangspunkt der Diskussionen sind zumeist statistische Befunde. Als äußerst problematisch gilt die unbereinigte Entgeltlücke (der sog. Gender Pay Gap), die hierzulande 23 Prozent $^{1}$ beträgt. Davon lassen sich nach Angaben des Statistischen Bundesamtes zwei Drittel „strukturell“ erklären, so dass eine bereinigte Entgeltlücke von acht Prozent bleibt. ${ }^{2}$ Eine noch geringere Differenz besteht nach Berechnungen des arbeitgebernahen Instituts der Deutschen Wirtschaft: Nur vier Prozent Lohneinbuße müssten Frauen in Kauf nehmen, wenn sie kindbedingte Erwerbsunterbrechungen möglichst kurz hielten. ${ }^{3}$ Der Berliner Tagesspiegel berichtete daraufhin auf der Titelseite: „Frauen verdienen fast so viel wie Männer“. ${ }^{4}$ Im öffentlichen Dienst Ostdeutschlands tritt das Phänomen der Verdienstdifferenz zwischen den Geschlechtern gar nicht auf, stellt das BMFSFJ in einer Studie fest. ${ }^{5}$

Angesichts der schwer nachvollziehbaren Berechnungsmethoden bleibt oft unklar, was die statistischen Ergebnisse genau bedeuten. Vielfach wird nach eindeutigen Aussagen gesucht, inwieweit Frauen bei der Bezahlung diskriminiert werden. Denn dies würde gegen das Grundgesetz und das Allgemeine Gleichbehandlungsgesetz (AGG) verstoßen. Arbeitgeber(innen) und Tarifparteien müssten eine solche Benachteiligung beseitigen; dies ist für sie Pflicht, nicht nur Kür. Häufig gestellte Fragen lauten daher: Inwieweit deutet die Entgeltlücke von 23 Prozent auf Benachteiligung von Frauen hin? Steckt nur der unerklärte Rest von acht Prozent den Rahmen für Entgeltdiskriminierung ab? Bezogen auf Ost-West-Unterschiede: Ist im öffentlichen Sektor Ostdeutschlands schon „alles im grünen Bereich “, da dort keine Verdienstdifferenz festgestellt wird? Und bezogen auf Unterschiede in der EU: Wie ist zu erklären, dass Italien nur einen Gender Pay Gap in Höhe von 4,9 Prozent hat? (Siehe hierzu auch die Erläuterungen im Kasten auf S. 11.) Und nicht zuletzt interessiert auf der betrieblichen Ebene: Wie müssten Entgeltberichte aussehen, um geschlechtsbezogene Entgeltdiskriminierungen sichtbar zu machen?

Die Antwort auf die Frage nach der Aussagekraft des Gender Pay Gap könnte kurz ausfallen: Weder die unbereinigte Entgeltdifferenz noch der statistisch unerklärte Rest sagen etwas über das Ausmaß von Entgeltdiskriminierung aus. In den gesamtwirtschaftlichen Analysen der Entgeltlücke spielen gleichstellungsrechtliche Kategorien keine Rolle. Der Gender Pay Gap beansprucht nicht, ein Indikator für Entgeltdiskriminierung zu sein. Vielmehr ist er für die Europäische Kommission einer der strukturellen Indikatoren, die zur Überwachung der europäischen Strategie für Wachstum und Beschäftigung herangezogen werden.
In diesem Beitrag sollen die Unterschiede zwischen einer statistischen und rechtlichen Analyse von „Entgelt(un)gleichheit“ ausführlicher erläutert werden. Anhand von fünf Beispielen soll illustriert werden, was Statistiken zeigen und was sie verbergen können. Abschließend wird der Frage nachgegangen, zu welchen politischen Strategien und Maßnahmen die unterschiedlichen Zugänge zum Problem der Entgeltungleichheit führen. Dies wirft die Frage auf: Reicht eine Politik der Verringerung der statistischen Entgeltdifferenz aus oder ist zusätzlich eine Politik der Antidiskriminierung erforderlich?

\section{Rechtliche Entgeltgleichheit oder nur gleich hohes Entgelt?}

Die ausgewählten statistischen Beispiele sind auf der betrieblichen Ebene angesiedelt, da sich Entgeltdiskriminierung nicht auf der gesamtwirtschaftlichen Ebene ermitteln lässt. Dies ergibt sich aus den Definitionen von unmittelbarer und mittelbarer Diskriminierung: 6

(1) Eine unmittelbare Geschlechtsdiskriminierung liegt vor, „wenn eine Person“ aufgrund ihres Geschlechts eine weniger günstige Behandlung erfährt als eine andere Person „in einer vergleichbaren Situation“ erfährt, erfahren hat oder erfahren würde. Mit der vergleichbaren Situation einer Frau und eines Mannes ist insbesondere die konkrete Arbeitssituation gemeint, da der Rechtsgrundsatz der Entgeltgleichheit darauf abstellt, dass gleiches Entgelt für gleiche oder gleichwertige Arbeit zu zahlen ist. ${ }^{7}$ Diese detaillierten arbeitsbezogenen Informationen, bei denen es zum Beispiel um feine Unterschiede im Anforderungsniveau der Arbeitsplätze geht, stehen auf gesamtwirtschaftlicher Ebene nicht zur Verfügung.

(2) Eine mittelbare Geschlechtsdiskriminierung liegt vor, wenn dem Anschein nach neutrale „Vorschriften, Kriterien oder Verfahren“ Personen wegen des Geschlechts gegenüber anderen Personen in besonderer Weise benachteiligen können, es sei denn, diese sind durch ein rechtmäßiges Ziel sachlich gerechtfertigt und zur Zielerreichung angemessen und erforderlich. Diese Definition verweist auf diskriminierende Regelungen, z.B. in Tarifverträgen oder Betriebsvereinbarungen. Die Analyse solcher Bestimmungen ist im Rahmen von gesamtwirtschaftlichen Statistiken nicht möglich. Auf betrieblicher Ebene stehen jedoch alle Informationen zur Prüfung von Entgeltdiskriminierung zur Verfügung.

\footnotetext{
1 Vgl. Statistisches Bundesamt, Pressemitteilung Nr. 384 vom 25.10.2010

2 Ebd.

3 Vgl. Institut der Deutschen Wirtschaft, Pressemeldung vom 9.10.2010, <http://www.iwkoeln.de/Publikationen/iwd/Archiv/ tabid/122/articleid/30645/Default.aspx> (Zugriff: 7.2.2011)

4 Tagesspiegel vom 10.11.2010.

5 Vgl. Studie des BMFSFJ: Der Verdienstunterschied zwischen Frauen und Männern im Öffentlichen Bereich und in der Privatwirtschaft, 2009, S. 14.

6 Vgl. \$3 Abs. 1 und 2 AGG und Art. 22 Abs. 1 der Richtlinie 2006/54/EG.

7 Vgl. Art. 4 der Richtlinie 2006/54/EG und § 8 Abs. 2 AGG.
} 


\section{Beispiel 1:}

\section{Statistische Entgeltdifferenz 10,7 Prozent - keine Entgeltdiskriminierung}

In einem Filialbetrieb arbeiten zehn Frauen und sechs Männer. Das monatliche Durchschnittseinkommen der Frauen beträgt 2.500 Euro, das der Männer 2.800 Euro. Es besteht eine Entgeltdifferenz in Höhe von 10,7 Prozent. Dieses Ergebnis sagt noch nichts über die Ursachen des geringeren Einkommens der Frauen und über den gleichstellungspolitischen Handlungsbedarf des Unternehmens aus. Der Unterschied könnte (teilweise) durch geschlechtsbezogene Diskriminierung verursacht sein, er könnte aber auch auf kulturellen und arbeitsmarktbezogenen Ungleichheitslagen im Geschlechterverhältnis beruhen, die personalpolitisch zementiert werden, jedoch keine Benachteiligung im rechtlichen Sinne darstellen. Um Entgeltdiskriminierung festzustellen, müssen die Tätigkeiten der Frauen und Männer in den Blick genommen werden. In diesem Fallbeispiel sind zehn Frauen und fünf Männer als Verkaufskräfte mit Kassierfunktion tätig. Sie verrichten die gleiche Tätigkeit und erhalten dafür monatlich brutto 2.500 Euro. In diesem Segment beträgt die Entgeltdifferenz 0 Prozent. Zusätzlich ist ein Filialleiter beschäftigt, der 4.300 Euro erhält. Dies erhöht den durchschnittlichen Bruttomonatsverdienst der Männer auf 2.800 Euro (Gesamtverdienst Männer 16.800 Euro ./. 6). Die Entgeltdifferenz beträgt nun 10,7 Prozent. Eine Entgeltdiskriminierung ist nicht erkennbar, denn gleiche Arbeit wird gleich bezahlt. Es gibt auch keine Verdachtsmomente für eine ungleiche Bezahlung gleichwertiger Arbeit, da die Tätigkeit der Filialleitung ein höheres Anforderungsniveau haben dürfte als die der Verkaufstätigkeit. Die Daten liefern auch keine Hinweise auf eine Beschäftigungsdiskriminierung, da dies eine Bewerbung einer weiblichen Verkaufskraft auf diese Stelle voraussetzt, über die ablehnend entschieden wurde. ${ }^{8}$ Rechtlicher Handlungsbedarf besteht für den Arbeitgeber nicht; er könnte jedoch zukünftig die Stelle der Filialleitung mit einer Frau besetzen, um die Gleichstellung der Geschlechter zu fördern.

\section{Beispiel 2:}

\section{Statistische Entgeltdifferenz o Prozent - Entgeltdiskriminierung möglich}

In einem Unternehmen ist keine Entgeltdifferenz zwischen den Geschlechtern feststellbar. Die sechs Frauen erhalten genauso viel wie die 16 Männer, nämlich monatlich 2.600 Euro brutto (Männer monatlich insgesamt 41.600 Euro, Frauen 15.600 Euro). Um nachvollziehen zu können, wie dieses Ergebnis zustande kommt, wird die Tätigkeits- und Entgeltstruktur nach Geschlecht abgebildet (s. Tabelle 1).

Was zeigt die Tabelle? Was nicht?

Die untere Entgeltgruppe ist ausschließlich mit fünf Männern (31 Prozent aller Männer) besetzt; dies senkt deren Durchschnittsentgelt beträchtlich ab. Insgesamt verteilen sich die Tätigkeiten der Männer über die ganze Breite des Entgeltgefüges. Die Frauen sind dagegen überwiegend in den mittleren bis höheren Entgeltgruppen zu finden. Durch diese Beschäftigtenstrukturen erklärt sich das gleich hohe Verdienstniveau.

\begin{tabular}{|l|c|c|c|}
\hline Tätigkeiten & Männer & Frauen & $\begin{array}{c}\text { Bruttomonats- } \\
\text { Entgelt in Euro }\end{array}$ \\
\hline Wartungsarbeiten & 5 & - & 1.900 \\
\hline Kaufmännische Assistenz & 1 & 1 & 2.300 \\
\hline Kraftfahrer(in) & 1 & - & 2.300 \\
\hline Sekretär(in) & - & 1 & 2.300 \\
\hline Facharbeiter(in) & 5 & - & 2.600 \\
\hline $\begin{array}{l}\text { Sachbearbeitung } \\
\text { Personal, Einkauf) }\end{array}$ & - & 3 & 2.600 \\
\hline $\begin{array}{l}\text { Facharbeiter(in) } \\
\text { mit Anleitungsfunktion }\end{array}$ & 3 & - & 3.200 \\
\hline Buchhaltung & 1 & - & 3.200 \\
\hline Bauingenieur(in) & & 1 & 4.900 \\
\hline
\end{tabular}

- Tabelle 1: Betriebliche Tätigkeits- und Entgeltstruktur nach Geschlecht

Hinsichtlich der Besetzung der Tätigkeiten zeigt sich weitgehend das traditionelle Muster: gewerblich und technisch orientierte Tätigkeiten werden von Männern, Angestelltentätigkeiten - mit Ausnahme der kaufmännischen Assistenztätigkeit - von Frauen wahrgenommen. Die höchste Entgeltgruppe ist von einem Mann besetzt.

Aus den Bruttomonatsentgelten kann weder geschlussfolgert werden, dass die kaufmännische Assistentin und der kaufmännische Assistent gleiches Entgelt für gleiche Arbeit erhalten, noch dass die Tätigkeiten der übrigen Frauen diskriminierungsfrei entlohnt werden. Dies zeigen die Beispiele 3 und 4.

\section{Beispiel 3:}

\section{Gleiches Gesamtbruttoentgelt bei gleicher Arbeit - Entgeltdiskriminierung zehn Prozent}

Wird lediglich das Gesamtbruttoentgelt von Frauen und Männern mit gleicher Arbeit betrachtet, bleibt eine etwaige Entgeltdiskriminierung unsichtbar, wie das Beispiel der kaufmännischen Assistentin und ihres männlichen Kollegen zeigt (s. Tabelle 1). Beide erhalten insgesamt jeweils 2.300 Euro brutto im Monat, die Entgeltdifferenz beträgt 0 Prozent. Die nähere Aufschlüsselung des Gesamtbetrages macht deutlich, dass er sich aus zwei Entgeltbestandteilen zusammensetzt: dem anforderungsbezogenen Grundentgelt (Grundstufe oder Einstiegsentgelt der Entgeltgruppe) und der Stufenzulage, mit der die Berufserfahrung honoriert wird (s. Tabelle 2).

Erst jetzt wird erkennbar, dass die Frau für die gleiche Arbeit monatlich 200 Euro weniger erhält als ihr Kollege. Dies weist auf eine unmittelbare Entgeltdiskriminierung aufgrund des Geschlechts hin. Die diskriminierungsbedingte Entgeltdifferenz beträgt zehn Prozent. Darüber hinaus wäre zu prüfen, ob die Einstufungen in die Stufen der Entgeltgruppe diskriminierungsfrei erfolgt sind. Ein Verstoß gegen das AGG könnte auch bei diesem Entgeltbestandteil vorliegen, wenn beispielsweise die Frau mehrere Jahre Berufserfahrung bei einem frü-

8 Vgl. hierzu auch BAG-Urteil v. 22.7.2010, 8 AZR 1012/o8. 


\begin{tabular}{|l|c|}
\hline Mann \\
\hline Kaufmännische Assistenz & 2.200 Euro \\
\hline Grundentgelt & 100 Euro \\
\hline Stufenzulage Stufe 1 & 2.300 Euro \\
\hline Gesamtbruttoentgelt & 2.000 Euro \\
\hline Frau & 300 Euro \\
\hline Kaufmännische Assistenz & 2.300 Euro \\
\hline Grundentgelt & \\
\hline Stufenzulage Stufe 3 &
\end{tabular}

$\Delta$ Tabelle 2: Gleich hohes Bruttoentgelt von Frau und Mann bei gleicher Arbeit

heren Arbeitgeber gesammelt hätte, diese jedoch bei der Einstellung nicht berücksichtigt würden. ${ }^{9}$ Diskriminierend wäre es auch, wenn die Frau zu Beginn der Elternzeit bereits die Stufe 4 ihrer Entgeltgruppe erreicht hätte, nach Rückkehr aus der Elternzeit jedoch in die Stufe 3 zurückgestuft worden wäre.

Kommentar aus rechtlicher Sicht:

- Die Entgeltbestandteile sind getrennt zu prüfen, da sonst die Diskriminierungsquelle nicht erkennbar ist und nicht beseitigt werden kann. Der EuGH stellte bereits 1990 fest: „In der Frage des gleichen Entgelts für Männer und Franen ist eine echte Durchschaubarkeit, die eine wirksame Kontrolle erlaubt, nur gewährleistet, wenn der Grundsatz des gleichen Entgelts für jeden einzelnen Bestandteil des den männlichen oder den weiblichen Arbeitnehmern gezablten Entgelts und nicht umfassend für die Gesamtheit der diesen beiden Arbeitnehmergruppen gewährten Vergütungen $z u$ beachten ist. " 10

- Der (die) Arbeitgeber(in) muss gleiche Arbeit gleich bezahlen, auch wenn die Frau weniger gefordert hat. Dies ergibt sich aus dem Rechtsgrundsatz des gleichen Entgelts für gleiche Arbeit. Erhält die Frau weniger, stellt dies eine Entgeltdiskriminierung dar. Mit Bescheidenheit in Verhandlungen kann ungleiches Entgelt daher nicht gerechtfertigt werden. Daraus folgt: Verantwortlich für die Beseitigung dieser Entgeltdifferenz ist nicht die einzelne Frau, sondern der (die) Arbeitgeber(in).

- In ökonomisch-statistischen Analysen wird die Entgeltdifferenz u.a. mit kindbedingten Unterbrechungszeiten von Frauen wegen Elternzeit erklärt. Denn in humankapitaltheoretischer Sicht stellen diese Zeiten eine Entwertung von Humankapital dar, die „Lohnstrafen“ nach sich zieht. Da das Gleichstellungsrecht in diesen Analysen regelmäßig außer Acht bleibt, wird nicht dafür sensibilisiert, dass eine Rückstufung nach der Elternzeit eine Diskriminierung darstellt. ${ }^{11}$ Die neue Elternzeit-Richtlinie 2010/18/EG formuliert in $\mathbb{5}$ Absatz 2: „Die Rechte, die der Arbeitnehmer zu Beginn des Elternurlaubs erworben hatte oder dabei war zu erwerben, bleiben bis zum Ende des Elternurlaubs be- stehen. Im Anschluss an den Elternurlaub finden diese Rechte mit den Änderungen Anwendung, die sich aus den nationalen Rechtsvorschriften, Tarifverträgen und/oder Gepflogenheiten ergeben."

\section{Beispiel 4: \\ Gleiche Eingruppierung unterschiedlicher Arbeit - Entgeltdiskriminierung möglich}

In Unternehmen und öffentlichen Verwaltungen sind oft verschiedenste Tätigkeiten von Frauen und Männern derselben Entgeltgruppe zugeordnet. Eine Statistik, die lediglich Durchschnittsentgelte der Frauen und der Männer einer Entgeltgruppe ausweist, lässt Entgeltdiskriminierung noch nicht erkennen. Denn aus der gleichen Eingruppierung inhaltlich unterschiedlicher Tätigkeiten kann nicht geschlossen werden, dass diese tatsächlich gleichwertig sind. Dies kann am Beispiel der Tätigkeiten „Sekretär(in)“ und „Kraftfahrer(in)“ illustriert werden (s. Tabelle 3). Beide Beschäftigte sind in die Entgeltgruppe 2 eingruppiert, beide erhalten ein Grundentgelt von 2.100 Euro (Grundstufe).

\begin{tabular}{|l|l|}
\hline $\begin{array}{l}\text { Sekretär(in) } \\
\text { Frau }\end{array}$ & Kraftfahrer(in) \\
Mann
\end{tabular}

A Tabelle 3: Gleiche Eingruppierung bei unterschiedlicher Arbeit

Für die Beurteilung der Gleichwertigkeit der Tätigkeiten ist entscheidend, ob die Tätigkeiten geschlechtsneutral bewertet wurden. Geschlechtsneutral bedeutet:

- Die Tätigkeiten müssen nach denselben Kriterien bewertet werden. ${ }^{12}$

- Diese Kriterien müssen „die Art der zu verrichtenden Arbeit" objektiv berücksichtigen und die Tätigkeiten ihrem „Wesen“" nach entlohnen ${ }^{13}$ und

- diskriminierungsfrei ausgelegt und angewendet werden.

- Das „System beruflicher Einstufung“ muss so beschaffen sein, dass Diskriminierungen aufgrund des Geschlechts ausgeschlossen werden. ${ }^{14}$ Hierunter fallen alle vom (von der) Arbeitgeber(in) angewendeten Verträge und Vertragsteile, an deren Aushandlung die gleichen Parteien beteiligt waren. ${ }^{15}$

Hätten die Tarifparteien - oder im Falle einer betrieblichen Regelung die Betriebsparteien - wesentliche Anforderungen oder Belastungen außer Acht gelassen, die sich an frauen- oder männerdominierte Tätigkeiten im Geltungsbereich der Regelung stellen, könnte dies zu einer diskriminierenden Unterbewertung dieser Tätigkeiten führen. Diesbezüglich wird schon

9 Auch wegen der europarechtlichen Freizügigkeit und der ethnischen Herkunft, vgl. EuGH v. 30.9.2003 - C 244/01 (Köbler).

10 EuGH v. 17.5.1990-Rs C-262/88 (Barber), Leitsatz 4.

11 Leider auch nicht seitens des BMFSFJ, vgl. BMFSFJ 2010, Ressortbericht 2010 - Verringerung des Verdienstabstandes zwischen Männern und Frauen, Berlin, S. 9 f.

12 Vgl. Art. 4 der Richtlinie 2006/54/EG.

13 Vgl. EuGH v. 1.7.1986 - Rs 237/85 (Rummler).

14 Vgl. Art. 4 der Richtlinie 2006/54/EG.

15 Vgl. EuGH v. 27.10.1993-Rs C-127/92 (Enderby). 
seit Langem bemängelt, dass bei der Bewertung von Dienstleistungstätigkeiten im öffentlichen Sektor „psycho-soziale Anforderungen“ bei der Arbeitsbewertung nicht bewertet werden. ${ }^{16}$

Kommentar aus rechtlicher Sicht:

- Das Wesen mittelbarer Entgeltdiskriminierung ist statistisch nicht erfassbar, da die Statistik nur die bestehende Bewertung abbilden kann, die ja gerade hinterfragt werden soll. Sie kann mit zwei Methoden sichtbar gemacht werden: erstens durch einen Check der geltenden Arbeitsbewertungs- und Eingruppierungsregelungen, der systematisch danach fragt, ob die oben genannten rechtlichen Anforderungen an eine diskriminierungsfreie Arbeitsbewertung erfüllt sind ${ }^{17}$. Zweitens können mittels eines diskriminierungsfreien Systems der Arbeitsbewertung die Vergleichstätigkeiten von Frau und Mann neu bewertet werden. ${ }^{18}$ Hierzu bedarf es Informationen über die Aufgaben und Anforderungen der jeweiligen Tätigkeiten.

- Die Eingruppierung der „Sekretär(in)“ in die Entgeltgruppe 2 könnte nicht allein durch Unterbewertung, sondern auch durch eine fehlerhafte betriebliche Eingruppierungsentscheidung verursacht sein. Wenn die Tätigkeit der Frau bei der Einstellung zu niedrig eingruppiert worden wäre, könnte eine unmittelbare Entgeltdiskriminierung vorliegen.

\section{Beispiel 5: \\ Bereinigte Entgeltdifferenz neun Prozent - Entgeltdiskriminierung nicht nur in diesem Teil möglich}

Die Bundesregierung empfiehlt Unternehmen, mit „Logib-D“ (Lohngleichheitsinstrument des Bundes - Deutschland“) die betriebliche Entgeltdifferenz zwischen den Geschlechtern zu ermitteln und sie mit vorgegebenen Strukturmerkmalen zu bereinigen. ${ }^{19}$ Ein im Internet veröffentlichtes fiktives Rechenbeispiel zeigt, welche Daten ein Entgeltbericht nach Logib-D enthalten könnte: ${ }^{20}$

Die unbereinigte durchschnittliche Entgeltdifferenz beträgt 22,6 Prozent. Diese Differenz wird damit erklärt, dass Frauen - mit gleichen Ausbildungsjahren wie Männer durchschnittlich

- mit gleichen potentiellen Erwerbsjahren

- mit gleichen Dienstjahren

- aufgrund des Geschlechts $-4,5$ Prozent $-2,0$ Prozent $-1,4$ Prozent - 9,0 Prozent weniger verdienen als Männer. Es wird zusätzlich berechnet, inwieweit Frauen und Männer ein Anforderungsniveau ${ }^{21}$ und eine berufliche Stellung 22 innehaben, die ihrem Humankapital (Ausbildung, potentielle Erwerbsjahre, Dienstjahre) entspricht.

Da rechtliche Kategorien bei dieser Analyse keine Rolle spielen, erfährt der (die) Arbeitgeber(in) nicht, inwieweit seine (ihre) Entgeltpraxis gleichheitswidrig ist. Er (sie) wird auch nicht dafür sensibilisiert, dass eine Entgeltdifferenzierung nach Ausbildungsjahren, potentiellen Erwerbsjahren und Dienstjahren Diskriminierung verursachen und tarifrechtlich problematisch sein kann. ${ }^{23}$ Es finden sich in dieser Software auch keine Hinweise darauf, dass Entgeltkürzungen nach Wiedereinstieg in den Beruf potentiell diskriminierend sind. Da die bei Logib-D verwendeten Erklärungsfaktoren selbst Diskriminierungspotential enthalten, sagt auch eine um diese Faktoren bereinigte Entgeltdifferenz nichts über Entgeltdiskriminierung aus. Selbst wenn der unerklärte Rest 0 Prozent betrüge, wäre Entgeltdiskriminierung nicht auszuschließen.

Bleibt noch die Frage, inwieweit die Analyseergebnisse für eine gleichstellungsorientierte Personalpolitik genutzt werden können. Hierzu wären insbesondere Informationen über Qualifikationspotentiale von Frauen dienlich. Hätte zum Beispiel eine Sachbearbeiterin ein abgeschlossenes betriebswirtschaftliches Studium und sogar einige Jahre Berufserfahrung in einer entsprechenden Tätigkeit gesammelt, könnte sie auch für besser bezahlte (Führungs-)Positionen in Frage kommen. Mit Logib-D werden jedoch nur die Zeiten der Ausbildung, Erwerbserfahrung und Betriebszugehörigkeit erfasst, nicht deren fachlich-inhaltliche Ausrichtung.

\section{Fazit und Schlussfolgerungen für politische Strategien}

Wie die Beispiele gezeigt haben, besteht kein systematischer Zusammenhang zwischen einer bereinigten oder unbereinigten Entgeltdifferenz und dem Ausmaß von Entgeltdiskriminierung. Es kann allenfalls das Rechtsprinzip des „gleichen Entgelts für gleiche Arbeit" statistisch geprüft werden, und dies auch nur unter drei Voraussetzungen:

(1) angewendet wird die Methode des direkten Vergleichs,

(2) es werden Männer und Frauen mit gleicher Tätigkeit verglichen und

(3) es wird nur das anforderungsbezogene Grundentgelt betrachtet das die Eingruppierung bestimmt.

Das Rechtsprinzip des ,gleichen Entgelts für gleichwertige Arbeit" kann nicht statistisch geprüft werden. Alle anderen Entgeltbestandteile sind getrennt entsprechend ihrer jeweiligen Begründungs- und Bewertungslogik zu prüfen.

Statistische Analysen, die humankapitaltheoretischen Annahmen folgen, setzen strategisch an Frauen und Männern als Individuen an. Die politische Botschaft lautet direkt oder indirekt: Frauen müssten ihr Humankapital auf das Niveau der Männer bringen. Das heißt: gleich lange Erwerbserfahrung und Betriebszugehörigkeitszeiten durch möglichst kurze oder

16 Vgl. Krell, Gertraude/Carl, Andrea Hilla/Krehnke, Anna (2001): Diskriminierungsfreie Bewertung von (Dienstleistungs-)Arbeit. Hrsg. von der Gewerkschaft ÖTV, Stuttgart.

17 Vgl. Instrument „Regelungs-Check zum anforderungsbezogenen Grundentgelt“ im Rahmen von „eg-check.de“.

18 Zum Beispiel mit einem „Paarvergleich zur Feststellung der Gleichwertigkeit“ im Rahmen von eg-check.de.

19 Vgl. 〈http://www.logib-d.de/DE/o1_Home/home_node.html〉 (Zugriff: 10.1.2011)

20 Vgl. Daten entnommen aus <http://www.logib-d.de/DE/o1_Home/ home_node.html> (Zugriff: 10.1.2011), fiktiver Ergebnisbericht (1), S. 9, Stand Dezember 2010.

21 Anforderungsniveau: wird zusammengefasst zu sechs Stufen, bildet nicht die Entgeltgruppen ab.

22 Berufliche Stellung: wird zusammengefasst zu sechs Stufen.

23 Vgl. EuGH v. 17.10.1989 - Rs 109/88 (Danfoss) sowie Winter, Regine, Diskriminierungsfreie(re) Entgeltgestaltung, in: Geschlechtergerechtigkeit, Festschrift für Heide Pfarr, 2010, S. 325 f. 


\section{Italien auf Platz 1: Wie ist die Entgeltlücke von nur 4,9 Prozent zu erklären?}

Im Vergleich der EU-Mitgliedstaaten belegt Italien mit einer Entgeltlücke von 4,9 Prozent den ersten Platz. Dies wird mit dem sogenannten „Sample Selection Bias“, einer statistischen Verzerrung durch Stichprobenselektion, erklärt: Italien gehört wie Malta zu den EU-Staaten, in denen die Frauenerwerbsquote sehr gering ist und nur wenige, jedoch hoch qualifizierte Frauen in die Stichprobe eingehen. Ihnen steht auf Seiten der Männer die ganze Bandbreite an Qualifikationsniveaus gegenüber, so die Ökonomin Friederike Maier. Dies gelte jedoch nicht für alle Länder, die die vorderen Plätze belegen, wie etwa Slowenien mit einem Gender Pay Gap von 8,5 Prozent oder Belgien mit neun Prozent. Quelle: Maier, Friederike (2008): Determinanten des Gender Pay Gap im internationalen Vergleich, Berlin, <http://www.genderkompetenz.info/ veranstaltungen/genderlectures/2008_10_27_hu> (Zugriff: 11.1.2011). bessere Betreuungsmöglichkeiten für Kinder wirken unterstützend und erleichtern die Vereinbarkeit von Familie und Beruf. So wichtig diese Maßnahmen auch sind: Sie richten sich überwiegend an Mütter und Frauen, die nach Führungspositionen streben. Und sie können Entgeltdiskriminierung nicht beseitigen, weder für diesen Kreis noch für alle anderen Arbeitnehmerinnen. Hierzu wäre eine Politik der Antidiskriminierung erforderlich. Diese würde jene Akteursgruppen ansprechen, die für die Festlegung der Arbeitsentgelte verantwortlich sind: einzelne Arbeitgeber(innen), Tarif- und Betriebsparteien. Sie würde darauf zielen, die betriebliche Entgeltpraxis und tarifliche Regelungen auf Entgeltdiskriminierung zu überprüfen. An geeigneten Prüfmethoden mangelt es zwischenzeitlich nicht mehr. ${ }^{24}$ „Gesetze helfen nur dann, wenn jemand kontrolliert, ob sie eingehalten werden “, fordert die Verbraucherministerin Ilse Aigner mit Blick auf die Kontrolle der Banken. ${ }^{25}$ Dies gilt auch für das gesetzliche Diskriminierungsverbot beim Arbeitsentgelt. Es bedarf verbindlicher gesetzlicher Regeln, wie sie bereits in anderen europäischen Ländern bestehen. ${ }^{26}$

\footnotetext{
24 Vgl. 〈www.eg-check.de〉 (Zugriff: 10.1.2011).

25 Der Tagesspiegel vom 28.12.2010, S. 15.

26 Z.B. Schweden, vgl. hierzu: Newsletter zur Entgeltgleichheit Nr. 4, hrsg. von Karin Tondorf und Andrea Jochmann-Döll, www.karin-tondorf.de.
}

\section{Aktuelle Pressemitteilungen und Stellungnahmen}

\section{Pressemitteilungen}

11-07 djb begrüßt EuGH-Urteil zu Unisex-Tarifen in der Privatversicherung (März 2011)

11-06 Wir können das?! (März 2011)

11-05 "Ohrfeige" für den Bundesgerichtshof: Bundesverfassungsgericht erklärt "Dreiteilungsmethode" für verfassungswidrig (Februar 2011)

11-04 Wochenendlektüre für MdBs: "Aktionärinnen fordern Gleichberechtigung" djb ruft 622 Bundestagsabgeordnete zur Unterstützung auf und überreicht ihnen die Projektstudie 2010 (Februar 2011)

11-03 Neue Wege in der Gleichstellungspolitik djb begrüßt den Ersten Gleichstellungsbericht der Bundesregierung (Januar 2011)

11-02 Refrain: „Sag mir wo die Frauen sind“. Die Aktion des djb „Aktionärinnen fordern Gleichberechtigung “ geht in die zweite Runde (Januar 2011)

11-01 Die zweite Chance nutzen und mit der Hartz-Reform die gleichberechtigte Teilhabe in der Gesellschaft fördern (Januar 2011)

10-31 Aktionärinnen fordern Gleichberechtigung. Das Projektergebnis 2010 - Präsentation und Diskussion der Studie am 1.12.2010 in Berlin (Dezember 2010)
10-30 Juristinnen fragen - Politikerinnen antworten. djbVeranstaltung zum Thema Entgelt(un)gleichheit am 25.11.2010, Berlin (November 2010)

10-29 Darf es ein bisschen mehr sein? Zwei Richterinnen für das Bundesverfassungsgericht gewählt (November 2010)

\section{Stellungnahmen}

11-01 Zum Referentenentwurf eines Gesetzes zur Stärkung der Recht von Opfern sexuellen Missbrauchs (StORMG) (Februar 2011)

10-14 Stellungnahme zu dem Gesetz zur Ermittlung von Regelbedarfen und zur Änderung des Zweiten und Zwölften Buches Sozialgesetzbuch (BT-Drs. 17/4304) sowie zu den Anträgen BT-Drs. 17/2934, 17/3435 sowie 17/3058 anlässlich der Anhörung im Ausschuss für Arbeit und Soziales des Deutschen Bundestages am Montag, den 22. November 2010 (November 2010)

10-13 Stellungnahme in dem Verfahren BVerfG 1 BvL 20/09 zu den Vorlagebeschlüssen des LSG München L $1 \mathrm{R}$ 204/09 und S 4 R 679/08 zur Verfassungsmäßigkeit der Regelung der Erziehungsrente in $\$ 47$ SGB VI (September 2010) 